\title{
Erratum
}

Monatsschr Kinderheilkd 2022 170 (Suppl 1): S30-S31

https://doi.org/10.1007/s00112-021-01221-w

Online publiziert: 12 . Juli 2021

๑) Der/die Autor(en) 2021

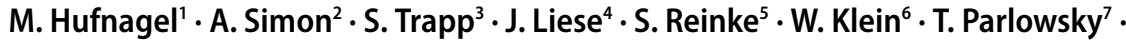
J. Pfeil ${ }^{8} \cdot$ H. Renk ${ }^{9} \cdot$ R. Berner ${ }^{10} \cdot$ J. Hübner ${ }^{11} \cdot$ S. Kummer ${ }^{12} \cdot$ R. Tillmann ${ }^{13}$

${ }^{1}$ Abteilung für Pädiatrische Infektiologie und Rheumatologie, Zentrum für Kinder- und Jugendmedizin, Universitätsklinik Freiburg, Freiburg, Deutschland; ${ }^{2}$ Klinik für Pädiatrische Onkologie und Hämatologie, Universitätsklinikum des Saarlandes, Homburg/Saar, Deutschland; ${ }^{3}$ Praxis für Kinder- und Jugendmedizin, Bremen, Deutschland; ${ }^{4}$ Kinderklinik und Poliklinik, UniversitätskinderklinikWürzburg, Würzburg, Deutschland; ${ }^{5}$ Klinik für Kinder- und Jugendmedizin, Clemenshospital, Münster, Deutschland; ${ }^{6}$ Praxis für Kinder- und Jugendmedizin, Augsburg, Deutschland; ' ${ }^{7}$ Praxis für Kinder- und Jugendmedizin, Lübeck, Deutschland; ${ }^{8}$ Praxis für Kinder- und Jugendmedizin, Schwaigern, Deutschland; ${ }^{9}$ Klinik für Kinder- und Jugendmedizin, Universitätsklinikum Tübingen, Tübingen, Deutschland; ${ }^{10} \mathrm{Klinik}$ und Poliklinik für Kinder- und Jugendmedizin, Universitätsklinikum Dresden, TU Dresden, Dresden, Deutschland; "Kinderklinik und Kinderpoliklinik, Dr. von Haunersches Kinderspital, München, Deutschland; ${ }^{12}$ Praxis für Kinder- und Jugendmedizin, Bielefeld, Deutschland; ${ }^{13}$ Praxis für Kinder- und Jugendmedizin, Bielefeld, Deutschland

\section{Erratum zu: Antibiotische Standardtherapie häufiger Infektionskrankheiten in der ambulanten Pädiatrie}

\section{Empfehlungen der Arbeitsgemeinschaft Antibiotic Stewardship ambulante Pädiatrie (ABSaP) der Deutschen Gesellschaft für Pädiatrische Infektiologie (DGPI), des Berufsverbandes der Kinder- und Jugendärzte (BVKJ) und der Bielefelder Initiative AnTiB}

\section{Erratum zu:}

Monatsschr Kinderheilkd 2020

https://doi.org/10.1007/s00112-020-

01011-w

Der Artikel Antibiotische Standardtherapie häufiger Infektionskrankheiten in der ambulanten Pädiatrie. Empfehlungen der Arbeitsgemeinschaft Antibiotic Stewardship ambulante Pädiatrie (ABSaP) der Deutschen Gesellschaft für Pädiatrische Infektiologie (DGPI), des Berufsverbandes der Kinder- und Jugendärzte (BVKJ) und der Bielefelder Initiative AnTiB von

M. Hufnagel $\cdot$ A. Simon $\cdot$ S. Trapp $\cdot$ J. Liese.S. Reinke $\cdot$ W. Klein.T. Parlowsky. J. Pfeil $\cdot H$. Renk $\cdot$ R. Berner $\cdot J$. Hübner.S. Kummer.R. Tillmann wurde ursprünglich Online First ohne „Open Access" auf der Internetplattform des Verlags publiziert. Nach der Veröffentlichung in Bd. 169 Heft 4 pp. 258-265 hatten sich die Autoren für eine „Open Access"-Veröffentlichung entschieden. Das Urheberrecht des Artikels wurde deshalb in (c) The Author(s) 2020 geändert.

\section{Korrespondenzadresse}

Prof. Dr. M. Hufnagel

Abteilung für Pädiatrische Infektiologie und Rheumatologie, Zentrum für Kinder- und Jugendmedizin, Universitätsklinik Freiburg Mathildenstr. 1, 79106 Freiburg, Deutschland markus.hufnagel@uniklinik-freiburg.de

Funding. Open Access funding enabled and organized by Projekt DEAL.

Open Access. Dieser Artikel wird unter der Creative Commons Namensnennung 4.0 International Lizenz veröffentlicht, welche die Nutzung, Vervielfältigung, Bearbeitung, Verbreitung und Wiedergabe in jeglichem Medium und Format erlaubt, sofern Sie den/die ursprünglichen Autor(en) und die Quelle ordnungsgemäß nennen, einen Link zur Creative Commons Lizenz beifügen und angeben, ob Änderungen vorgenommen wurden. 
Die in diesem Artikel enthaltenen Bilder und sonstiges Drittmaterial unterliegen ebenfalls der genannten Creative Commons Lizenz, sofern sich aus der Abbildungslegende nichts anderes ergibt. Sofern das betreffende Material nicht unter der genannten Creative Commons Lizenz steht und die betreffende Handlung nicht nach gesetzlichen Vorschriften erlaubt ist, ist für die oben aufgeführten Weiterverwendungen des $\mathrm{Ma}$ terials die Einwilligung des jeweiligen Rechteinhabers einzuholen.

Weitere Details zur Lizenz entnehmen Sie bitte der Lizenzinformation auf http://creativecommons.org/ licenses/by/4.0/deed.de. 